\title{
Understanding the Pathobiology of Head and Neck Squamous Cell Carcinoma
}

\author{
Chia-Cheng Li $\cdot$ Sook-Bin Woo
}

Published online: 11 July 2014

(C) Springer International Publishing AG 2014

\begin{abstract}
Head and neck squamous cell carcinoma (HNSCC) is one of the leading cancers in the world, although wide geographical variations do exist. HNSCC can be subcategorized into conventional HNSCC and HPV-associated HNSCC, exhibiting distinct clinical and histopathologic features. Awareness of the risks of smoking has fortunately contributed to the decreasing incidence of conventional HNSCC in the USA. However, the prevalence of HPV-associated HNSCC in the USA has been significantly increasing. Much progress has been made in the research of development and progression of HNSCC. In this article, we review the current concepts of the pathobiological mechanisms of HNSCC.
\end{abstract}

Keywords Head and neck squamous cell carcinoma . Pathobiology $\cdot$ High-risk HPV

C.-C. Li $(\bowtie) \cdot$ S.-B. Woo

Department of Oral Medicine, Infection, and Immunity, Harvard

School of Dental Medicine, Boston, MA 02115, USA

e-mail: chia-cheng_li@hsdm.harvard.edu

S.-B. Woo

e-mail: swoo@rics.bwh.harvard.edu

C.-C. $\mathrm{Li}$

Division of Genetics, Department of Medicine, Brigham and

Women's Hospital, Boston, MA 02115, USA

S.-B. Woo

Division of Oral Medicine and Dentistry, Brigham and Women's

Hospital, Boston, MA 02115, USA

S.-B. Woo

Center for Oral Pathology, StrataDx, Lexington, MA 02421, USA

\section{Introduction to Head and Neck Squamous Cell Carcinoma}

Cancer, a major cause of mortality, is a global health issue, and squamous cell carcinoma (SCC) of the head and neck constitutes $90 \%$ of all cancer cases in the oral and oropharyngeal regions [1]. There is a wide geographic variation. Based on a study of cancer incidence in 2008, an estimated 263,900 new SCC cases only from the oral cavity and lips were newly diagnosed worldwide $(170,900$ cases in males, $64.8 \%$; and 93,000 cases in females, $35.2 \%$ ), with 128,000 deaths [2]. Oral SCC is the $8^{\text {th }}$ leading cancer in men in the USA with an approximate annual incidence of 40,000 new cases and 8,000 deaths [3-7]. However in countries such as India and Pakistan, where there is a high prevalence of areca nut use, oral SCC represents the most common cancer in men $[2,8]$.

Multiple factors contribute to the initiation and progression of head and neck squamous cell carcinoma (HNSCC). Usage of areca nut, alcohol, and tobacco are well-recognized risk factors for SCC, both for the oral cavity proper and the oropharyngeal region [9]. Human papillomavirus (HPV) infection on the other hand, stands out as a risk factor for oropharyngeal SCC, with increasing incidence noted within the last 15 years $[2,10]$. HPV-positive HNSCC exhibits a better prognosis than HPV-negative HNSCC for specific anatomic locations (e.g., tonsils), with a five-year survival rate of $89 \%$ and $65 \%$, respectively [11]. Moreover, the median survival period is significantly longer in HPV-positive oropharyngeal SCC (91 months) compared to HPV-negative ones (76 months) [11]. In addition to the aforementioned exogenous risk factors, certain hereditary conditions, such as Fanconi anemia and Li-Fraumeni Syndrome, are predisposed to HNSCC [12]. The mainstay of treatment for HNSCC is surgery with adjuvant radiotherapy or chemo-radiotherapy for high stage disease with accompanying morbidity. The overall 
five-year survival rate of conventional HNSCC is approximately $60 \%[3,7]$.

Targeted therapies are now used increasingly to manage cancers and improve survival rate. Such therapies specifically target crucial proteins within key pathways in the development of cancer. Although much progress has been made in the study of the development and progression of HNSCC, many specific mechanisms are still unclear. This article reviews the current concepts underlying the pathobiology of HNSCC.

\section{HNSCC Initiation}

\section{Cancer Stem Cells (Cancer Initiating Cells)}

HNSCCs cause significant morbidity and mortality, and may recur after surgical excision and adjuvant chemotherapy and radiotherapy [13]. This clinical fact has led to the cancer stem cell (CSC) model that hypothesizes the existence of a distinct cell population within HNSCC tumors, which may be resistant to the conventional therapy. HNSCC contains cellular heterogeneity, within which a developmental hierarchy exists [14]. CSCs or cancer initiating cells, consist of less than $10 \%$ of the cells in an HNSCC tumor, and are able to self-renew and to aberrantly differentiate into heterogeneous cancer cell populations [15]. Identification and characterization of these CSCs will help us gain more insight in cancer biology, predict the tumor behavior, and develop better treatments to efficiently target this cell population.

Currently, flow cytometry, in vitro clonal trial, and serial transplantations are some methodologies used to detect CSCs. Several CSC-like markers (e.g., CD44; Bmi1; aldehyde dehydrogenase, ALDH; and CD133) have been identified, and the labeled cells demonstrated robust tumorigenic activity after serial transplantations [15-17]. Furthermore, evidence suggests that the existence of these labeled cells in oral SCC is associated with a poorer prognosis [18]. CD44 is associated with MMP9 activity [19], and the up-regulated expression of CD44 can potentially be utilized to determine tumor invasiveness and aggressiveness [20]. Bmil (B-cell specific Moloney murine leukemia virus insertion site 1) is a transcription repressor for cell senescence, implicated in the self-renewal of stem cells [21]. Bmil is highly expressed in the CD44 $4^{+}$cell population sorted from oral SCC tumors, suggesting a potential role within CSCs [15]. ALDH labels a highly tumorigenic cell subset within the $\mathrm{CD} 44^{+}$population [16]. Compared to the $\mathrm{CD} 133^{-}$human oral SCC cells, the CD133 overexpressed cell population demonstrates stemness, invasiveness, and tumorigenic activity [17]. CD133 coordinates with the Src signaling pathway and contributes to epithelial-mesenchymal transition [17]. While multiple markers have been identified, and have shed light on the etiologic role of CSCs, the detailed signaling mechanisms are not yet fully understood.

\section{Field Cancerization}

The development of cancer through the entire upper aerodigestive tract is due to the accumulation of multiple genetic abnormalities via a multi-step process whereby cells in a particular tissue/organ are genetically altered but histologically normal, predating the development of neoplasia or coexisting with malignant cells [22-24]. As such, it is possible that precancerous lesions that already carry mutations may not even be visible clinically or abnormal histologically. This concept of "field cancerization" was proposed by Slaughter in 1953 to explain the high frequency of development of synchronous or metachronous HNSCCs $[25,26]$.

Microscopically, HNSCCs often have wider horizontal spread than infiltrative growth ("lateral cancerization") [24, 25]. In excised HNSCC specimens with clinically "clear" margins, hyperkeratotic, hyperplastic, or abnormally atrophic epithelium is often seen adjacent to the cancerous lesions, indicating that the lateral field is already "cancerized" [25, 26]. Within the "normal appearing" epithelium approximating the cancer, chromosomal aberrations (e.g., loss of heterzogosity, microsatellite alterations), and genetic/ epigenetic mutations have been detected [26, 27]. Loss of heterzygosity $(\mathrm{LOH})$ is the loss of one allele of a gene (e.g., tumor suppressor gene), such that if the remaining allele containing the other copy of the same gene becomes inactivated by a point mutation, this will result in the affected individual being more susceptible to cancer. $\mathrm{LOH}$ at the chromosomes 17p, 3p, 9p, 8p, 18q, and 11q has been associated with HNSCCs, and the order of occurrence as a progression model of HNSCCs has been proposed [26]. However, due to the lack of proper control and longitudinal follow-up, this model is not universally accepted. Nevertheless, it is likely as with cancers elsewhere in the body, that as a result of cumulative molecular events, CSCs transform to gain clonality and a selective proliferation advantage. The detailed pathologic mechanisms of HNSCCs will be discussed in the following sections.

\section{Pathobiologic Mechanisms of HNSCC}

To initiate a cancer, accumulated genetic and epigenetic alterations within an otherwise normal cell allows it to acquire the capabilities of becoming neoplastic and malignant. Six hallmarks of cancer were proposed by Weinberg and Hanahan in 2011 to conceptualize the initiation, progression, and dissemination of malignancies $[28,29]$. Gene mutations leading to the up-regulation of oncogenes or down-regulation of tumor suppressor genes are seen in many cancers, including HNSCC. Epigenetic modifications are heritable changes that regulate gene activity through adjusting the structure and function of 
chromatin, without affecting DNA code sequences, by processes such as DNA hypermethylation and post-translational histone modifications [30]. DNA hypermethylation, interrupting the binding of transcription factors at promoter regions and silencing of tumor suppressor genes (e.g., p16), is commonly detected in HNSCCs $(50 \%-73 \%)$ [31, 32]. In addition, DNA hypermethylation of CDH1, MGMT, and DAPK in HNSCCs have been reported [31-33]. Interestingly, tissues adjacent to cancerous lesions also exhibit hypermethylation of these genes, suggesting methylation as an early molecular event in carcinogenesis [31,34]. However, there is no direct evidence indicating that the level of DNA methylation correlates with recurrence or infiltrative growth of HNSCCs.

In addition to genetic and epigenetic changes, microRNAs (miRNAs) are also involved in the initiation and progression of HNSCCs. MiRNAs are short, single-stranded, 18-23 nucleotide-long, non-coding RNA molecules, which repress the expression of its target genes post-transcriptionally [35]. The mechanisms of miRNA biogenesis and function were described in detail previously [36]. Altered expression of miRNAs may be caused by transcriptional dysregulation of miRNAs, chromosomal aberration, epigenetic changes, single nucleotide polymorphisms (SNPs), or defects in the miRNAs processing machinery, and is closely associated with the initiation and progression of malignancies [37]. Approximately fifty miRNAs have been linked with HNSCC. A full list of HNSCC miRNAs can be found in the database HeNeCan miRs, and is available at http://tarmir.rgcb.res.in/henecan/ [38]. Some HNSCC-related miRNAs, known as oncomirs, possess oncogenic capacity by several mechanisms, such as dysregulating key cell cycle checkpoints to promote unchecked proliferation (e.g., miR-106b) [39, 40], interrupting the crosstalk between tumor growth factor-beta (TGF- $\beta$ ) and Myc pathways (e.g., miR-106b-25/miR-17-92 cluster), which leads to cell cycle dysregulation and resistance to apoptosis [41], or interrupting apoptosis through the PI3K/PTEN/AKT pathway (e.g., miR-21) [40].

\section{HNSCC Initiates with Limitless Proliferation of Epithelial Cells}

A cancer cell exhibits uncontrolled proliferation, and invades the surrounding tissue, suggesting that the underlying pathobiological mechanisms involve dysregulation of proliferative signaling pathways, inhibition of growth suppressors, resistance to cell death, and maintenance of replicative immortality [29].

\section{Dysregulation of Proliferative Signaling Pathways}

Growth factors stimulate cell proliferation via binding to the surface receptors of target cells, activating kinases with transduction into the nuclei to further activate the intracellular signaling cascade. Epidermal growth factor (EGF) is critical for the initiation and progression of HNSCC. In addition to growth factor ligand-dependent pathways, activation of downstream signaling pathways (e.g., phosphatidylinositol-3kinase, PI3K/AKT pathway) alone may also lead to the dysregulation of cell proliferation.

EGF receptor (EGFR), a member of the ErbB/Her family, is a transmembrane receptor tyrosine kinase [42]. Once bound with the ligand (e.g., EGF and TGF $\alpha$ ), EGFRs activate several downstream signaling cascades, including Ras/Raf/mitogen-activated protein kinase (MAPK), PI3K/AKT, mammalian target of rapamycin (mTOR), Janus kinase (Jak)/signal transducer and activator of transcription (STAT), and protein kinase C (PKC) pathways [43]. The EGFR pathway regulates cell proliferation and survival, and is associated with tumor invasion, metastasis, and angiogenesis [44]. Aberrant activation of EGFR pathway is prevalent in HNSCC [45, 46], which may present as amplification of EGFR, mutation of EGFR, or overproduction of ligands [47]. EGFR variant III (EGFRvIII), a truncation mutated variant, leads to conformational activation of the EGFR without ligand binding or receptor overproduction [48]. Cells with EGFRvIII demonstrated higher resistance to chemotherapy [48].

A monoclonal antibody to EGFR, cetuximab, is the first molecularly targeted therapy to receive US Food and Drug Administration (FDA) approval for treating HNSCC [49]. Cetuximab improved overall survival when used to manage locally or regionally advanced and recurrent/metastatic HNSCCs $[50,51]$. Based on the National Comprehensive Cancer Network (NCCN) guidelines, cetuximab plus 5-fluorouracil and platinum (cisplatin or carboplatin) is a category 1 treatment option for patients with unresectable or recurrent/ metastatic non-nasopharyngeal HNSCC [52]. Other targeted therapies include tyrosine kinase inhibitors (e.g., lapatinib, dacomitinib, and afatinib) and mTOR inhibitors (e.g., temsirolimus and everolimus) which are currently being used in clinical trials for HNSCC [49].

\section{Inhibition of Growth Suppressors and Resistance of Cell Death}

Cancer cells gain limitless replicative potential through altering the cell cycle, within which Retinoblastoma (RB) and TP53, as tumor suppressor genes, play important roles (as will be noted in HPV-related lesions below) [53]. There are two critical check points of the cell cycle in the G1 and G2 phases that are regulated by complexes of cyclins and cyclindependent kinases (CDKs). RB protein, a tumor suppressor and a critical regulator in $\mathrm{G}_{1} / \mathrm{S}$ cell cycle progression, typically binds to and inactivates transcription factor $\mathrm{E} 2 \mathrm{~F}$ and the ensuing signaling cascade. When RB is phosphorylated, E2F is released to activate the transcription of c-Myc, cyclin A, and p21/WAF-1, so that cell cycle proceeds [54]. Loss of function 
or expression of RB results in uncontrolled cell proliferation. TP53 is a tumor suppressor gene, playing a critical role in cell cycle progression, cell differentiation and apoptosis. TP53 mutation commonly occurs at a hot spot region at codon 245 and codon 248 [55]. Somatic mutations of TP53 were found in $50 \% \sim 80 \%$ of HNSCC cases [56-58], and disruptive mutation of TP53 is strongly associated with decreased overall survival rate [58].

Microenvironment Enhances the Progression and Invasion of HNSCC

In addition to the genetic/epigenetic dysregulation within cancer cells, recent studies suggest that the connective tissue may significantly contribute to the progression and invasion of HNSCC [59]. The natural boundary of an epithelium is maintained by normal interactions with the underlying connective tissue and basement membrane [60]. When neoplastic processes begin, the microenvironment demonstrates increased microvasculature, modified extracellular matrix (ECM) deposited by cancer-associated fibroblasts (CAFs), and infiltration of inflammatory cells. The key findings of HNSCC microenvironment are summarized below.

\section{Induction of Angiogenesis}

The rapid growth of solid tumors, such as HNSCC, results in hypoxia of the tissue, and induces angiogenesis [61]. Hypoxia activates the secretion of vascular endothelial growth factors (VEGFs), mediated by hypoxia-inducible transcription factor $1 \alpha(\mathrm{HIF}-1 \alpha)$ and $2 \alpha$ (HIF-2 $\alpha)$ within tumor cells $[62,63]$. In addition to hypoxia, growth factors (e.g., EGF) or their receptors (e.g., EGFR), cytokines (e.g., IL-6), and protein products of activated oncogenes or mutated tumor suppressor genes, may also regulate the expression level of VEGF [63, 64]. VEGF-A, one of the VEGF family members, is a $45-\mathrm{kDa}$ homodimeric glycoprotein with different isoforms and diverse range of angiogenic activities [65]. VEGF-A is particularly critical in tumor angiogenesis, and has been found overexpressed in both HNSCC cell lines and specimens [66-68]. The overexpression of VEGF-A is correlated with poor prognosis and lymph node metastasis in HNSCC [68-70]. Cancer cells as well as surrounding stromal cells, such as fibroblasts, produce VEGF and encourage neovasculaturization through the VEGF/VEGF receptor (VEGFR) axis, especially VEGFR2, which triggers multiple downstream signaling pathways, leading to the survival, migration, and differentiation of endothelial cells [63]. VEGFR2-mediated survival, proliferation, and migration of endothelial cells are further enhanced by activating pathways of PI3K, MAPK, and focal adhesion kinase, respectively [71, 72]. In addition, VEGF also enhances microvasculature permeability [73]. Therefore, it may provide a path for metastatic dissemination of malignant cells. The calcium-dependent pathway, Akt signaling pathway and Erk1/2 pathway increase the level of nitric acid, cGMP and prostaglandins, also suggesting they play a role in increasing vessel permeability $[74$, 75]. However, the detailed regulatory mechanisms need further elucidation.

VEGF overexpression has been shown as a critical component regulating angiogenesis in HPV16-positive cervical SCC [76]. E6 viral oncoprotein binds to the promoter region of VEGF and activates the expression level of VEGF [77]. Moreover, in HPV16-positive tumor cells, VEGF can enhance cell proliferative activity through up-regulating EGFR, while in the HPV16-negative cervical cancer, up-regulation of mutated EGFR plays a major role of driving cell proliferation [78]. However, the relationship between HPV16 infection and VEGF expression remains controversial in oropharyngeal SCC $[79,80]$. In conventional HNSCC, up-regulation of VEGF is correlated with poor clinical outcome, but this correlation becomes weaker in HPV16-positive HNSCC [80]. Further research on angiogenesis of HPV16-positive and negative HNSCC is required to elucidate the underlying mechanisms.

\section{Interplay Between Cancer Cells and Stromal Cells}

In addition to the genetic/epigenetic dysregulation within cancer cells, stromal cells, also known as CAFs (cancerassociated fibroblasts), may be critical in the progression and invasion of HNSCC. CAFs demonstrate myofibroblastic features with cytoplasmic accumulation of $\alpha$-smooth muscle actin ( $\alpha$-SMA), and have a role in synthesizing ECM, such as type I collagen [81]. In HNSCC, CAFs are frequently associated with dense collagen deposition and stromal desmoplasia [82]. Stromal $\alpha$-SMA positivity was observed in $100 \%$ of the conventional SCC, but not in the nonneoplastic or adjacent uninvolved stroma. Together with loss of CD34, gain of stromal $\alpha$-SMA positivity links myofibroblasts and tumor microenvironment with tumor invasion [83]. Up-regulation of $\alpha$-SMA and integrin- $\alpha 6$ in CAFs is shown to be correlated with invasiveness and poor prognosis of oral SCC [84]. Integrin- $\alpha 6$ is critical for cell-cell adhesion and cell-ECM interaction as well as downstream signaling cascades that regulate cell cycle.

CAFs also produce variable cytokines, such as CXCL12 and matrix metalloproteinases (MMPs), which promote cell motility and invasiveness of HNSCC. The CXCL12/CXCR4 axis, first discovered in the trafficking of hematopoietic stem cells to the bone marrow, plays a key role in modulating tumor microenvironment, as well as up-regulating expression of MMP9 and HIF-1 $\alpha$ [85, 86]. CXCL12, also called SDF-1 (stromal cell-derived factor-1), is one of the chemokines that binds to a transmembrane G-protein coupled receptor, CXCR4 [85]. 
CAFs-derived CXCL12 induces invasion and dispersing of CXCR4-overexpressed oral SCC cells, and is strongly associated with regional lymph node metastasis of oral SCC [87-89]. MMPs (e.g., MMP-2 and MMP-9), a group of proteolytic enzymes that degrade and remodel ECM components, are regulated by multiple signaling pathways (such as EGFR), which are commonly overexpressed in HNSCC [90]. Up-regulation of MMPs in HNSCC is associated with increased metastatic rate and poor prognosis [91, 92]; therefore, the underlying mechanisms are worthy of further investigation.

Epithelial-Mesenchymal Transition Contributes to the Invasion and Metastasis of HNSCC

Epithelial-mesenchymal transition (EMT) is a fundamental biological process in embryonic morphogenesis that plays a critical role in cancer development and progression, and is considered a crucial event linked to tumor invasion and metastasis. The classical EMT features in cancer cells include loss of cell polarity and E-cadherin, as well as acquisition of mesenchymal features, namely spindle morphology, motile phenotype, and expression of mesenchymal markers (e.g., vimentin and SMA) [93].

TGF- $\beta$ pathway is a major player triggering EMT in HNSCC [94]. In TGF- $\beta$ signal transduction, dimerized ligands bind to type I and type II receptor heterodimers to phosphorylate and activate the transcription factor Smad. Smad proteins consist of three functional classes, namely the receptor-regulated Smad (R-Smad), the comediator Smad (Co-Smad), and the inhibitory Smad (ISmad) [95]. R-Smad (Smad1, Smad2, Smad3, Smad5 and Smad8) is directly phosphorylated by type I receptor kinases. TGF- $\beta$ signaling pathway selectively activates Smad2/3 [95]. The dimerized phosphorylated R-Smads then form a regulatory complex with the Co-Smad (Smad4), and translocate to the nucleus to activate the expression of downstream genes. On the other hand, the I-Smads (Smad6 and Smad7) compete with R-Smads for interaction with the Co-Smad and type I receptor [95].

TGF- $\beta$ pathway also contributes to EMT through STAT3-mediated activation of Twist, Snail, and Slug $[96,97]$. Twist induces Bmi-1, and the two act cooperatively to down-regulate E-cadherin but up-regulate Ncadherin in HNSCC, which is referred to as a "cadherin switching" phenomenon [98]. Slug also contributes to cadherin switching in response of hypoxia-related HIF$1 \alpha$ expression, and is correlated with poor prognosis in HNSCC [99]. Emerging evidence suggests that EMT is a process of gaining "stemness" or becoming CSCs, which may contribute to the resistance to conventional therapies $[17,100]$.

\section{Emerging Technologies in HNSCC Research}

Massively parallel sequencing, also known as the nextgeneration sequencing, is a high-throughput sequencing technique to analyze the genomes of HNSCC thoroughly and efficiently. Two groups published their results back-to-back in Science in 2011, performing exome sequencing for both tumor DNA and the corresponding normal DNA from the same patient [101, 102]. In addition to previously described mutations in HNSCC (e.g., TP53, CDKN2A and PIK3CA), a novel mutation, NOTCH, is identified [103].

There are four members in the mammalian NOTCH receptor family, NOTCH $1-4$. When the NOTCH ligand binds to the NOTCH receptor, the intracellular domain translocates into the nucleus and activates downstream genes, such as NOTCH receptors, NOTCH ligands, cyclin D1 (CCND1), and MYC, which are critical for the cell cycle regulation [103]. In addition to TP53, NOTCH mutation is the second most common mutation in HNSCC, occurring in $10 \%-15 \%$ of the HNSCC cases [104]. In HNSCC, approximately $40 \%$ of the mutations in NOTCH1 were truncated gene products, suggesting that NOTCH1 may act as a tumor suppressor gene in HNSCC [101]. This novel approach provides us a comprehensive overview of the gene profiles of HNSCC, and contributes significantly to our understanding in cancer biology.

\section{HPV-Associated HNSCC}

HPV-associated HNSCC has been recognized as a clinically and biologically distinctive variant of SCC which occurs predominantly in the oropharynx of younger male patients (median age 56.9 years) [105]. HPV is an epitheliotropic, double-stranded DNA virus, with approximately 150 subtypes identified, of which 120 have been fully sequenced [106, 107]. High risk HPVs, mainly HPV16, are associated with $25.9 \%$ of the HNSCC cases, with the highest prevalence in oropharyngeal SCC (35\%-40\%) followed by laryngeal SCC $(24 \%)$ and oral SCC (23.5\%) [108, 109]. Moreover, the prevalence of high risk HPV in oropharyngeal SCC has significantly increased from $35 \%-40 \%$ before 2000 to $72.2 \%$ in 2005-2009 [110].

High risk HPVs induce carcinogenesis via two important viral oncoproteins, E6 and E7, which cause dysregulation of the cell cycle and apoptosis [106]. Viral protein E7 binds to $\mathrm{RB}$, and causes reduced expression of $\mathrm{RB}$ and overexpression of p16INK4a (a gene product of CDKN2A), leading to proliferation and cell cycle progression [107]. Viral protein E6 inhibits wild type TP53, a major factor for the G2 check point, through binding to and triggering of the ubiquitin-mediated degradation of TP53. This results in a compromised ability of the infected cells to engage cell cycle checkpoints and apoptotic responses. In HPV-associated HNSCC, mutated p53 is 
not expected in the tumor cells as compared with conventional HNSCC [111]. The molecular consequences of expressing E6 and E7 include interruption of p53-mediated apoptosis and unlimited proliferation. It may be due to the absence of p53 mutation that HPV-associated HNSCC demonstrates better prognosis compared to the HPV-negative HNSCC [112].

\section{Conclusion}

HNSCC is one of the leading cancers in the world associated with a high mortality rate. Multiple risk factors have been identified, including tobacco, alcohol, and areca nut consumption, as well as high risk HPV infection. Currently, surgical removal with adjuvant chemotherapy and radiotherapy is still the mainstay of treatment. Emerging technologies contribute to an understanding of the underlying pathobiological mechanisms and help in the development of personalized targeted therapy.

Acknowledgments The authors thank Dr. Peter Hammerman for the insightful discussions regarding genetic mutations in HNSCCs using genome-wide association study.

\section{Compliance with Ethics Guidelines}

Conflict of Interest Dr. Chia-Cheng Li and Dr. Sook-Bin Woo each declare no potential conflicts of interest.

Human and Animal Rights and Informed Consent This article does not contain any studies with human or animal subjects performed by any of the authors.

\section{References}

1. Warnakulasuriya S. Global epidemiology of oral and oropharyngeal cancer. Oral Oncol. 2009;45:309-16.

2. Jemal A et al. Global cancer statistics. CA Cancer J Clin. 2011;61: 69-90.

3. Jemal A, Siegel R, Xu J, Ward E. Cancer statistics, 2010. CA Cancer J Clin. 2010;60:277-300.

4. Siegel R, Ward E, Brawley O, Jemal A. Cancer statistics, 2011: the impact of eliminating socioeconomic and racial disparities on premature cancer deaths. CA Cancer J Clin. 2011;61:212-36.

5. Siegel R, Naishadham D, Jemal A. Cancer statistics, 2012. CA Cancer J Clin. 2012;62:10-29.

6. Siegel R, Naishadham D, Jemal A. Cancer statistics, 2013. CA Cancer J Clin. 2013;63:11-30.

7. Siegel R, Ma J, Zou Z, Jemal A. Cancer Statistics, 2014. CA Cancer J Clin. 2014.

8. Warnakulasuriya S. Living with oral cancer: epidemiology with particular reference to prevalence and life-style changes that influence survival. Oral Oncol. 2010;46:407-10.

9. de Camargo CM, de Souza DL, Curado MP. International incidence of oropharyngeal cancer: a population-based study. Oral Oncol. 2012;48:484-90

10. D'Souza G et al. Case-control study of human papillomavirus and oropharyngeal cancer. N Engl J Med. 2007;356:1944-56.
11. Ang KK et al. Human papillomavirus and survival of patients with oropharyngeal cancer. N Engl J Med. 2010;363: $24-35$.

12. van Monsjou HS, Wreesmann VB, van den Brekel MW, Balm AJ. Head and neck squamous cell carcinoma in young patients. Oral Oncol. 2013;49:1097-102.

13. Scully C, Bagan J. Oral squamous cell carcinoma: overview of current understanding of aetiopathogenesis and clinical implications. Oral Dis. 2009; 15:388-99.

14. Sayed SI et al. Implications of understanding cancer stem cell (CSC) biology in head and neck squamous cell cancer. Oral Oncol. 2011;47:237-43.

15. Prince ME et al. Identification of a subpopulation of cells with cancer stem cell properties in head and neck squamous cell carcinoma. Proc Natl Acad Sci U S A. 2007;104:973-8.

16. Clay MR et al. Single-marker identification of head and neck squamous cell carcinoma cancer stem cells with aldehyde dehydrogenase. Head Neck. 2010;32:1195-201.

17. Chen YS et al. CD133/Src axis mediates tumor initiating property and epithelial-mesenchymal transition of head and neck cancer. PLoS One. 2011;6:e28053.

18. Gonzalez-Moles MA, Scully C, Ruiz-Avila I, Plaza-Campillo JJ. The cancer stem cell hypothesis applied to oral carcinoma. Oral Oncol. 2013;49:738-46.

19. Aruffo A, Stamenkovic I, Melnick M, Underhill CB, Seed B. CD44 is the principal cell surface receptor for hyaluronate. Cell. 1990;61: 1303-13.

20. Joshua B et al. Frequency of cells expressing CD44, a head and neck cancer stem cell marker: correlation with tumor aggressiveness. Head Neck. 2012;34:42-9.

21. Park IK, Morrison SJ, Clarke MF. Bmi1, stem cells, and senescence regulation. J Clin Invest. 2004;113:175-9.

22. Califano $J$ et al. Genetic progression model for head and neck cancer: implications for field cancerization. Cancer Res. 1996;56: 2488-92.

23. Feller L, Lemmer J. Cell transformation and the evolution of a field of precancerization as it relates to oral leukoplakia. Int J Dent. 2011;2011:321750.

24. Angadi PV, Savitha JK, Rao SS, Sivaranjini Y. Oral field cancerization: current evidence and future perspectives. J Oral Maxillofac Surg. 2012;16:171-80.

25. Slaughter DP, Southwick HW, Smejkal W. Field cancerization in oral stratified squamous epithelium; clinical implications of multicentric origin. Cancer. 1953;6:963-8.

26. Braakhuis BJ, Tabor MP, Kummer JA, Leemans CR, Brakenhoff RH. A genetic explanation of Slaughter's concept of field cancerization: evidence and clinical implications. Cancer Res. 2003;63:1727-30.

27. Tabor MP et al. Persistence of genetically altered fields in head and neck cancer patients: biological and clinical implications. Clin Cancer Res. 2001;7:1523-32.

28. Hanahan D, Weinberg RA. The hallmarks of cancer. Cell. 2000;100:57-70.

29. Hanahan D, Weinberg RA. Hallmarks of cancer: the next generation. Cell. 2011;144:646-74.

30. Egger G, Liang G, Aparicio A, Jones PA. Epigenetics in human disease and prospects for epigenetic therapy. Nature. 2004;429: 457-63.

31. Kulkarni V, Saranath D. Concurrent hypermethylation of multiple regulatory genes in chewing tobacco associated oral squamous cell carcinomas and adjacent normal tissues. Oral Oncol. 2004;40:14553.

32. Kato $\mathrm{K}$ et al. Aberrant promoter hypermethylation of $\mathrm{p} 16$ and MGMT genes in oral squamous cell carcinomas and the surrounding normal mucosa. J Cancer Res Clin Oncol. 2006;132:735-43. 
33. Yeh KT et al. The correlation between $\mathrm{CpG}$ methylation on promoter and protein expression of E-cadherin in oral squamous cell carcinoma. Anticancer Res. 2002;22:3971-5.

34. Maruya $\mathrm{S}$ et al. Differential methylation status of tumor-associated genes in head and neck squamous carcinoma: incidence and potential implications. Clin Cancer Res. 2004;10:3825-30.

35. Lagos-Quintana M, Rauhut R, Lendeckel W, Tuschl T. Identification of novel genes coding for small expressed RNAs. Science. 2001;294:853-8.

36. Bartel DP. MicroRNAs: genomics, biogenesis, mechanism, and function. Cell. 2004;116:281-97.

37. Gorenchtein M, Poh CF, Saini R, Garnis C. MicroRNAs in an oral cancer context - from basic biology to clinical utility. J Dent Res. 2012;91:440-6.

38. Babu JM, Prathibha R, Jijith VS, Hariharan R, Pillai MR. A miRcentric view of head and neck cancers. Biochim Biophys Acta. 2011;1816:67-72.

39. Ivanovska I et al. MicroRNAs in the miR-106b family regulate $\mathrm{p} 21 /$ CDKN1A and promote cell cycle progression. Mol Cell Biol. 2008;28:2167-74.

40. Hui $\mathrm{AB}$ et al. Comprehensive MicroRNA profiling for head and neck squamous cell carcinomas. Clin Cancer Res. 2010;16:1129 39.

41. Petrocca F, Vecchione A, Croce CM. Emerging role of miR-106b25/miR-17-92 clusters in the control of transforming growth factor beta signaling. Cancer Res. 2008;68:8191-4.

42. Hynes NE, Lane HA. ERBB receptors and cancer: the complexity of targeted inhibitors. Nat Rev Cancer. 2005;5:341-54.

43. Choi S, Myers JN. Molecular pathogenesis of oral squamous cell carcinoma: implications for therapy. J Dent Res. 2008;87:14-32.

44. Reuter CW, Morgan MA, Eckardt A. Targeting EGF-receptorsignalling in squamous cell carcinomas of the head and neck. Br J Cancer. 2007;96:408-16.

45. Grandis JR, Tweardy DJ. Elevated levels of transforming growth factor alpha and epidermal growth factor receptor messenger RNA are early markers of carcinogenesis in head and neck cancer. Cancer Res. 1993;53:3579-84.

46. Ongkeko WM, Altuna X, Weisman RA, Wang-Rodriguez J. Expression of protein tyrosine kinases in head and neck squamous cell carcinomas. Am J Clin Pathol. 2005;124:71-6.

47. Kalyankrishna S, Grandis JR. Epidermal growth factor receptor biology in head and neck cancer. J Clin Oncol. 2006;24:2666-72.

48. Sok JC et al. Mutant epidermal growth factor receptor (EGFRvIII) contributes to head and neck cancer growth and resistance to EGFR targeting. Clin Cancer Res. 2006;12:5064-73.

49. Cohen RB. Current challenges and clinical investigations of epidermal growth factor receptor (EGFR)- and ErbB family-targeted agents in the treatment of head and neck squamous cell carcinoma (HNSCC). Cancer Treat Rev. 2014;40:567-77.

50. Bonner JA et al. Radiotherapy plus cetuximab for squamous-cell carcinoma of the head and neck. N Engl J Med. 2006;354:567-78.

51. Vermorken JB et al. Platinum-based chemotherapy plus cetuximab in head and neck cancer. N Engl J Med. 2008;359:1116-27.

52. National Comprehensive Cancer Network. NCCN clinical practice guidelines in oncology ${ }^{\mathrm{TM}}$. Head and Neck Cancers. V.2.2013 $<\mathrm{http}$ ://www.nccn.org/professionals/physician_gls/PDF/head-andneck.pdf $>$.

53. Kastan MB, Bartek J. Cell-cycle checkpoints and cancer. Nature. 2004;432:316-23.

54. Goodger NM, Gannon J, Hunt T, Morgan PR. Cell cycle regulatory proteins-an overview with relevance to oral cancer. Oral Oncol. 1997;33:61-73.

55. Somers KD et al. Frequent p53 mutations in head and neck cancer. Cancer Res. 1992;52:5997-6000.

56. van Houten VM et al. Mutated p53 as a molecular marker for the diagnosis of head and neck cancer. J Pathol. 2002;198:476-86.
57. Balz $\mathrm{V}$ et al. Is the p53 inactivation frequency in squamous cell carcinomas of the head and neck underestimated? Analysis of p53 exons 2-11 and human papillomavirus 16/18 E6 transcripts in 123 unselected tumor specimens. Cancer Res. 2003;63:1188-91.

58. Poeta ML et al. TP53 mutations and survival in squamous-cell carcinoma of the head and neck. N Engl J Med. 2007;357:2552-61.

59. Curry JM et al. Tumor microenvironment in head and neck squamous cell carcinoma. Semin Oncol. 2014;41:217-34.

60. Hill MW, Mackenzie IC. The influence of differing connective tissue substrates on the maintenance of adult stratified squamous epithelia. Cell Tissue Res. 1984;237:473-8.

61. Folkman J. Role of angiogenesis in tumor growth and metastasis. Semin Oncol. 2002;29:15-8.

62. Semenza GL. Targeting HIF-1 for cancer therapy. Nat Rev Cancer. 2003;3:721-32.

63. Hicklin DJ, Ellis LM. Role of the vascular endothelial growth factor pathway in tumor growth and angiogenesis. J Clin Oncol. 2005;23: 1011-27.

64. Kedar D, Baker CH, Killion JJ, Dinney CP, Fidler IJ. Blockade of the epidermal growth factor receptor signaling inhibits angiogenesis leading to regression of human renal cell carcinoma growing orthotopically in nude mice. Clin Cancer Res. 2002;8:3592-600.

65. Tischer $\mathrm{E}$ et al. The human gene for vascular endothelial growth factor. Multiple protein forms are encoded through alternative exon splicing. J Biol Chem. 1991;266:11947-54.

66. Salven P, Heikkila P, Anttonen A, Kajanti M, Joensuu H. Vascular endothelial growth factor in squamous cell head and neck carcinoma: expression and prognostic significance. Mod Pathol. 1997;10: 1128-33.

67. Shemirani B, Crowe DL. Head and neck squamous cell carcinoma lines produce biologically active angiogenic factors. Oral Oncol. 2000;36:61-6.

68. Oc P, Rhys-Evans P, Eccles SA. Expression of vascular endothelial growth factor family members in head and neck squamous cell carcinoma correlates with lymph node metastasis. Cancer. 2001;92(556-568).

69. Smith BD, Smith GL, Carter D, Sasaki CT, Haffty BG. Prognostic significance of vascular endothelial growth factor protein levels in oral and oropharyngeal squamous cell carcinoma. J Clin Oncol. 2000;18:2046-52.

70. Kyzas PA, Cunha IW, Ioannidis JP. Prognostic significance of vascular endothelial growth factor immunohistochemical expression in head and neck squamous cell carcinoma: a meta-analysis. Clin Cancer Res. 2005;11:1434-40.

71. Abedi H, Zachary I. Vascular endothelial growth factor stimulates tyrosine phosphorylation and recruitment to new focal adhesions of focal adhesion kinase and paxillin in endothelial cells. J Biol Chem. 1997;272:15442-51.

72. Veikkola T, Karkkainen M, Claesson-Welsh L, Alitalo K. Regulation of angiogenesis via vascular endothelial growth factor receptors. Cancer Res. 2000;60:203-12.

73. Dvorak HF, Brown LF, Detmar M, Dvorak AM. Vascular permeability factor/vascular endothelial growth factor, microvascular hyperpermeability, and angiogenesis. Am J Pathol. 1995;146: 1029-39.

74. Zachary I. Signaling mechanisms mediating vascular protective actions of vascular endothelial growth factor. Am J Physiol Cell Physiol. 2001;280:C1375-86.

75. Bates DO, Harper SJ. Regulation of vascular permeability by vascular endothelial growth factors. Vascul Pharmacol. 2002;39:22537.

76. Guidi AJ et al. Vascular permeability factor (vascular endothelial growth factor) expression and angiogenesis in cervical neoplasia. J Natl Cancer Inst. 1995;87:1237-45.

77. Lopez-Ocejo O et al. Oncogenes and tumor angiogenesis: the HPV16 E6 oncoprotein activates the vascular endothelial growth factor 
(VEGF) gene promoter in a p53 independent manner. Oncogene. 2000;19:4611-20.

78. Mathur RS, Mathur SP. Vascular endothelial growth factor (VEGF) up-regulates epidermal growth factor receptor (EGF-R) in cervical cancer in vitro: this action is mediated through HPV-E6 in HPVpositive cancers. Gynecol Oncol. 2005;97:206-13.

79. Fei J et al. Prognostic significance of vascular endothelial growth factor in squamous cell carcinomas of the tonsil in relation to human papillomavirus status and epidermal growth factor receptor. Ann Surg Oncol. 2009;16:2908-17.

80. Troy JD et al. Expression of EGFR, VEGF, and NOTCH1 suggest differences in tumor angiogenesis in HPV-positive and HPVnegative head and neck squamous cell carcinoma. Head Neck Pathol. 2013;7:344-55.

81. Serini G, Gabbiani G. Mechanisms of myofibroblast activity and phenotypic modulation. Exp Cell Res. 1999;250:273-83.

82. Wheeler SE et al. Enhancement of head and neck squamous cell carcinoma proliferation, invasion, and metastasis by tumorassociated fibroblasts in preclinical models. Head Neck. 2014;36: 385-92.

83. Paral KM, Taxy JB, Lingen MW. CD34 and alpha smooth muscle actin distinguish verrucous hyperplasia from verrucous carcinoma. Oral Surg Oral Med Oral Pathol Oral Radiol. 2014;117:477-82.

84. Lim KP et al. Fibroblast gene expression profile reflects the stage of tumour progression in oral squamous cell carcinoma. J Pathol. 2011;223:459-69.

85. Zlotnik A. Chemokines in neoplastic progression. Semin Cancer Biol. 2004;14:181-5.

86. Ishikawa $\mathrm{T}$ et al. Hypoxia enhances CXCR4 expression by activating HIF-1 in oral squamous cell carcinoma. Oncol Rep. 2009;21: $707-12$.

87. Uchida D et al. Possible role of stromal-cell-derived factor-1/ CXCR4 signaling on lymph node metastasis of oral squamous cell carcinoma. Exp Cell Res. 2003;290:289-302.

88. Delilbasi CB, Okura M, Iida S, Kogo M. Investigation of CXCR4 in squamous cell carcinoma of the tongue. Oral Oncol. 2004;40:154-7.

89. Ishikawa T et al. CXCR4 expression is associated with lymph-node metastasis of oral squamous cell carcinoma. Int J Oncol. 2006;28: 61-6.

90. Ohnishi Y, Inoue H, Furukawa M, Kakudo K, Nozaki M. Heparinbinding epidermal growth factor-like growth factor is a potent regulator of invasion activity in oral squamous cell carcinoma. Oncol Rep. 2012;27:954-8.

91. Makinen LK et al. Prognostic significance of matrix metalloproteinase-2, $-8,-9$, and -13 in oral tongue cancer. J Oral Pathol Med. 2012;41:394-9.

92. Koontongkaew S. The tumor microenvironment contribution to development, growth, invasion and metastasis of head and neck squamous cell carcinomas. J Cancer. 2013;4:66-83.

93. Thiery JP. Epithelial-mesenchymal transitions in tumour progression. Nat Rev Cancer. 2002;2:442-54.

94. Ikushima H, Miyazono K. TGFbeta signalling: a complex web in cancer progression. Nat Rev Cancer. 2010;10:415-24.
95. Shi Y, Massague J. Mechanisms of TGF-beta signaling from cell membrane to the nucleus. Cell. 2003;113:685-700.

96. Cho KH et al. STAT3 mediates TGF-beta1-induced TWIST1 expression and prostate cancer invasion. Cancer Lett. 2013;336:16773.

97. Liu RY et al. JAK/STAT3 signaling is required for TGF-betainduced epithelial-mesenchymal transition in lung cancer cells. Int J Oncol. 2014;44:1643-51.

98. Yang MH et al. Bmil is essential in Twist1-induced epithelialmesenchymal transition. Nat Cell Biol. 2010;12:982-92.

99. Zhang J, Cheng Q, Zhou Y, Wang Y, Chen X. Slug is a key mediator of hypoxia induced cadherin switch in HNSCC: correlations with poor prognosis. Oral Oncol. 2013;49:1043-50.

100. Zhang Z, Dong Z, Lauxen IS, Filho MS, Nor JE. Endothelial cellsecreted EGF induces epithelial to mesenchymal transition and endows head and neck cancer cells with stem-like phenotype. Cancer Res. 2014;74:2869-81.

101. Agrawal $\mathrm{N}$ et al. Exome sequencing of head and neck squamous cell carcinoma reveals inactivating mutations in NOTCH1. Science. 2011;333:1154-7.

102. Stransky $\mathrm{N}$ et al. The mutational landscape of head and neck squamous cell carcinoma. Science. 2011;333:1157-60.

103. Ranganathan $P$, Weaver KL, Capobianco AJ. Notch signalling in solid tumours: a little bit of everything but not all the time. Nat Rev Cancer. 2011;11:338-51.

104. Sun W et al. Activation of the NOTCH pathway in head and neck cancer. Cancer Res. 2014;74:1091-104.

105. Hoffmann $\mathrm{M}$ et al. Human papillomaviruses in head and neck cancer: 8 year-survival-analysis of 73 patients. Cancer Lett. 2005;218:199-206.

106. zur Hausen H. Papillomaviruses and cancer: from basic studies to clinical application. Nat Rev Cancer. 2002;2:342-50.

107. Rautava J, Syrjanen S. Biology of human papillomavirus infections in head and neck carcinogenesis. Head Neck Pathol. 2012;6 Suppl 1:S3-15.

108. Kreimer AR, Clifford GM, Boyle P, Franceschi S. Human papillomavirus types in head and neck squamous cell carcinomas worldwide: a systematic review. Cancer Epidemiol Biomarkers Prev. 2005; 14:467-75.

109. Leemans CR, Braakhuis BJ, Brakenhoff RH. The molecular biology of head and neck cancer. Nat Rev Cancer. 2011;11:9-22.

110. Mehanna $\mathrm{H}$ et al. Prevalence of human papillomavirus in oropharyngeal and nonoropharyngeal head and neck cancer-systematic review and meta-analysis of trends by time and region. Head Neck. 2013;35:747-55.

111. Wiest T, Schwarz E, Enders C, Flechtenmacher C, Bosch FX. Involvement of intact HPV16 E6/E7 gene expression in head and neck cancers with unaltered $\mathrm{p} 53$ status and perturbed $\mathrm{pRb}$ cell cycle control. Oncogene. 2002;21:1510-7.

112. Westra WH et al. Inverse relationship between human papillomavirus-16 infection and disruptive p53 gene mutations in squamous cell carcinoma of the head and neck. Clin Cancer Res. 2008;14:366-9. 